\title{
Patching retinal breaks with Seprafilm for treating retinal detachments in humans: 9 years of follow-up
}

${ }^{1}$ Department of Ophthalmology, Kurume University, School of Medicine, Kurume, Japan

${ }^{2}$ Arai Eye Clinic, Fukuoka, Japan

${ }^{3}$ Sueda Eye Clinic, Kurume, Japan

${ }^{4}$ Schepens Eye Research Institute, Harvard Medical School, Boston, MA, USA

Correspondence: M Haruta, Department of Ophthalmology, Kurume University, School of Medicine, 67 Asahi-machi, Kurume, Fukuoka 8300011, Japan

Tel: +81 94231 7574;

Fax: +81942370324.

E-mail: haruta_masatoshi@ med.kurume-u.ac.jp

Received: 31 August 2016 Accepted in revised form: 19 December 2016;

Published online:

27 January 2017

\begin{abstract}
Purpose To describe the long-term surgical outcomes of four patients treated for retinal detachment using Seprafilm as a novel technique.

Methods Retinal breaks in four eyes were covered with Seprafilm using a transvitreal approach after cataract surgery, pars plana vitrectomy, fluid-air exchange, and laser photocoagulation. Neither long-standing gas nor silicone oil was used. The patients were not instructed to maintain a specific head positioning postoperatively.

Results Successful retinal reattachment was achieved with a single surgery in all four eyes, and none developed proliferative vitreoretinopathy. The mean best-corrected visual acuity preoperatively and 9 years postoperatively were 20/97 and 20/33, respectively. The intraocular pressure increased several days postoperatively that lasted no longer than 2 weeks. Visual field defects either in the inferonasal or inferotemporal quadrant were detected postoperatively. The mean electroretinogram a- and b-wave amplitude ratios of the operated eyes to the fellow eyes were 0.68 and 0.64 preoperatively and 0.87 and 0.92 postoperatively, respectively. The mean corneal endothelial cell density was 2365 cells $/ \mathrm{mm}^{2}$ preoperatively and 2592 cells $/ \mathrm{mm}^{2}$ postoperatively.

Conclusion Covering retinal breaks with Seprafilm may promote retinal reattachment without gas tamponade and postoperative head positioning. The visual outcomes 9 years postoperatively showed no apparent adverse effects of intraocular application of Seprafilm. Eye (2017) 31, 776-780; doi:10.1038/eye.2016.329; published online 27 January 2017
\end{abstract}

Introduction

Rhegmatogenous retinal detachments (RRDs) continue to be important causes of visual loss
M Haruta ${ }^{1}$, M Arai ${ }^{2}$, J Sueda ${ }^{3}$, T Hirose $^{4}$ and $\mathrm{R}$ Yamakawa ${ }^{1}$ and blindness. Although recent vitreoretinal surgeries have high success rates with visual recovery, proliferative vitreoretinopathy (PVR) remains a major reason for surgical failure. ${ }^{1}$ The pathogenesis of PVR is associated with breakdown of the blood-retinal barrier and intravitreal dispersion of proliferating retinal pigment epithelial cells. ${ }^{2}$ Therefore, it appears reasonable to assume that we can prevent PVR by covering the bare retinal breaks with a biocompatible film that blocks intravitreal cellular migration. ${ }^{3}$ Furthermore, gas tamponade and postoperative head positioning may not be required if retinal breaks can be covered by a biocompatible film. ${ }^{3}$

Seprafilm Adhesion Barrier (Sanofi, Bridgewater, NJ, USA), a bioresorbable translucent membrane comprising sodium hyaluronate and carboxymethylcellulose, was developed to prevent adhesions after abdominal and pelvic surgeries. ${ }^{4,5}$ In vitro, Seprafilm adhered well to the retina and was impermeable to water. ${ }^{6}$ When Seprafilm was applied to patch the retinal breaks in an animal model, retinal reattachment occurred without a toxic or inflammatory reaction as assessed by retinal histology and electroretinography. ${ }^{7}$ The purpose of the current study was to determine whether we could cover retinal breaks with Seprafilm and to confirm long-term safety of intraocular Seprafilm application in humans.

\section{Subjects and methods}

This prospective study was performed at the Department of Ophthalmology of Kurume University Hospital, Japan. Four patients with a RRD underwent pars plana vitrectomy (PPV) and the retinal breaks were covered with Seprafilm between February 2006 and April 2006. Only retinal tears that could be covered with $5 \times 2 \mathrm{~mm}$ sheets of Seprafilm were included 
in the study. The institutional review board approved the study. All patients were informed about PPV performed with or without Seprafilm to repair the retinal detachments, and all patients provided written informed consent to proceed with application of Seprafilm.

Surgery was performed under local anesthesia. Phacoemulsification and intraocular lens implantation was performed on all the four phakic eyes to avoid cataract progression. After 20 gauge vitrectomy and fluid-air exchange, the subretinal fluid was removed using a backflush needle to reattach the retina, and the retinal breaks were treated with endophotocoagulation. A $5 \times 2 \mathrm{~mm}$ sheet of Seprafilm was delivered into the eye through one of the sclerotomy sites that was expanded to $3 \mathrm{~mm}$ wide, and Seprafilm was placed over the retinal breaks using intraocular forceps (see Supplementary Video 1, Supplementary Information). The tip of an intraocular lens injector was cut off and the tip then was used to deliver the Seprafilm into the eye. Neither longstanding gas nor silicone oil was used. The patients were not instructed to take a specific head positioning postoperatively.

Besides the immediate postoperative periods, patients were examined preoperatively and 1,3, and 6 months postoperatively. All four patients were further evaluated 9 years postoperatively. The preoperative and postoperative evaluations included measurement of the best-corrected visual acuity (BCVA) and intraocular pressure (IOP), slit-lamp biomicroscopy, dilated fundus examination, corneal endothelial specular microscopy, fundus photography, optical coherence tomography, electroretinography, and Goldmann visual-field examination. The electroretinogram (ERG) amplitudes of the maximal responses were measured after a minimal 30-min period of dark adaptation. The BCVA values were converted to the logarithm of the minimum angle of resolution visual acuities to calculate the mean BCVA values.

\section{Results}

Tables 1 and 2 show the clinical preoperative and postoperative data from the four patients. Figure 1 illustrates case 3 in which a 42-year-old male was treated for a RRD and Seprafilm was applied. On the first postoperative day, the Seprafilm was barely visible on fundus examination and $\sim 60-70 \%$ of the vitreous cavity was filled with air. The air was gone completely in a mean of 9.5 days. The Seprafilm was undetectable with a fundus examination 2 weeks postoperatively. Additional procedures performed during the 9 years postoperatively included laser photocoagulation in one eye (case 1) and posterior capsulotomy in two eyes (cases 1 and 4).

The retina remained reattached 1,3 , and 6 months and 9 years postoperatively in all eyes. During the follow-up period, a thin epiretinal membrane (case 1) and macular

Table 1 Clinical characteristics of rhegmatogenous retinal detachments

\begin{tabular}{|c|c|c|c|c|c|c|c|c|c|c|c|c|c|c|}
\hline Case & $\begin{array}{c}\text { Age } \\
\text { (years) }\end{array}$ & Gender & Eye & $\begin{array}{c}A L \\
(m m)\end{array}$ & $\begin{array}{l}\text { Spherical } \\
\text { equivalent } \\
\text { (diopters) }\end{array}$ & $\begin{array}{l}\text { Lens } \\
\text { status }\end{array}$ & $\begin{array}{l}\text { Symptom } \\
\text { duration }\end{array}$ & Macula & $\begin{array}{l}\text { Detached } \\
\text { retinal } \\
\text { quadrants }\end{array}$ & $\begin{array}{l}\text { Status of } \\
\text { retinal } \\
\text { detachment }\end{array}$ & $\begin{array}{l}\text { Break } \\
\text { type }\end{array}$ & $\begin{array}{l}\text { Numbers } \\
\text { of breaks }\end{array}$ & Size of breaks & $\begin{array}{c}\text { Break } \\
\text { position } \\
\text { (clock hours) }\end{array}$ \\
\hline 1 & 68 & M & $\mathrm{L}$ & 22.9 & 0.0 & Phakic & 8 Days & On & 1 & Bullous & Hole & 1 & $<1$ Clock hour & $7: 00$ \\
\hline 2 & 32 & F & $\mathrm{L}$ & 23.6 & -5.25 & Phakic & 2 Months & Off & 2 & Shallow & Holes & 2 & $<1$ Clock hour & $5: 00$ \\
\hline 3 & 42 & M & $\mathrm{L}$ & 26.8 & -7.0 & Phakic & 10 Days & Off & 2 & Bullous & Tear & 1 & $<1$ Clock hour & $3: 30$ \\
\hline 4 & 55 & $\mathrm{~F}$ & $\mathrm{~L}$ & 25.4 & -6.0 & Phakic & 7 Days & Off & 1 & Bullous & Tear & 1 & $<1$ Clock hour & $1: 00$ \\
\hline
\end{tabular}

Abbreviations: AL, axial length; F, female; L, left; M, male.

Table 2 Follow-up data

\begin{tabular}{|c|c|c|c|c|c|c|c|c|c|c|c|c|c|c|c|c|}
\hline \multirow[t]{2}{*}{ Case } & \multicolumn{5}{|c|}{$B C V A$} & \multicolumn{5}{|c|}{$I O P$} & \multicolumn{2}{|c|}{ a-Wave ratio } & \multicolumn{2}{|c|}{$b$-Wave ratio } & \multicolumn{2}{|c|}{ CECD } \\
\hline & Pre & $\begin{array}{l}1 \text { Mon } \\
\text { post }\end{array}$ & $\begin{array}{l}3 \text { Mon } \\
\text { post }\end{array}$ & $\begin{array}{l}6 \text { Mon } \\
\text { post }\end{array}$ & $\begin{array}{l}9 Y r \\
\text { post }\end{array}$ & Pre & $\begin{array}{l}1 \text { Mon } \\
\text { post }\end{array}$ & $\begin{array}{l}3 \text { Mon } \\
\text { post }\end{array}$ & $\begin{array}{l}6 \text { Mon } \\
\text { post }\end{array}$ & $\begin{array}{l}9 Y r \\
\text { post }\end{array}$ & Pre & $\begin{array}{l}9 Y r \\
\text { post }\end{array}$ & Pre & $\begin{array}{l}9 Y r \\
\text { post }\end{array}$ & Pre & $\begin{array}{l}9 Y r \\
\text { post }\end{array}$ \\
\hline 1 & $20 / 22$ & $20 / 40$ & $20 / 29$ & $20 / 29$ & $20 / 20$ & 18 & 21 & 20 & 17 & 17 & 0.55 & 0.74 & 0.42 & 0.77 & 2016 & 1916 \\
\hline 2 & $20 / 222$ & $20 / 286$ & $20 / 222$ & $20 / 222$ & $20 / 133$ & 14 & 11 & 9 & 13 & 11 & 0.71 & 0.82 & 0.78 & 0.84 & 2695 & 3676 \\
\hline 3 & $20 / 133$ & $20 / 40$ & $20 / 22$ & $20 / 22$ & $20 / 22$ & 16 & 18 & 16 & 15 & 19 & 0.99 & 0.91 & 1.05 & 0.93 & 2232 & 2451 \\
\hline 4 & $20 / 133$ & $20 / 29$ & $20 / 29$ & $20 / 22$ & $20 / 20$ & 16 & 14 & 13 & 16 & 19 & 0.46 & 1.02 & 0.32 & 1.13 & 2518 & 2326 \\
\hline Mean & $20 / 97$ & $20 / 60$ & $20 / 45$ & $20 / 42$ & $20 / 33$ & 16 & 16 & 15 & 15 & 17 & 0.68 & 0.87 & 0.64 & 0.92 & 2365 & 2592 \\
\hline
\end{tabular}

a-Wave ratio, electroretinogram a-wave amplitude ratio of operated eye to fellow eye; BCVA, best-corrected visual acuity; b-Wave ratio, electroretinogram $\mathrm{b}$-wave amplitude ratio of operated eye to fellow eye; CECD, corneal endothelial cell density (cells $/ \mathrm{mm}^{2}$ ); IOP, intraocular pressure (mm Hg); Mon, month; post, postoperative; pre, preoperative; $\mathrm{Yr}$, year.

The BCVA values were converted to the logarithm of the minimum angle of resolution visual acuities to calculate the mean BCVA values. 


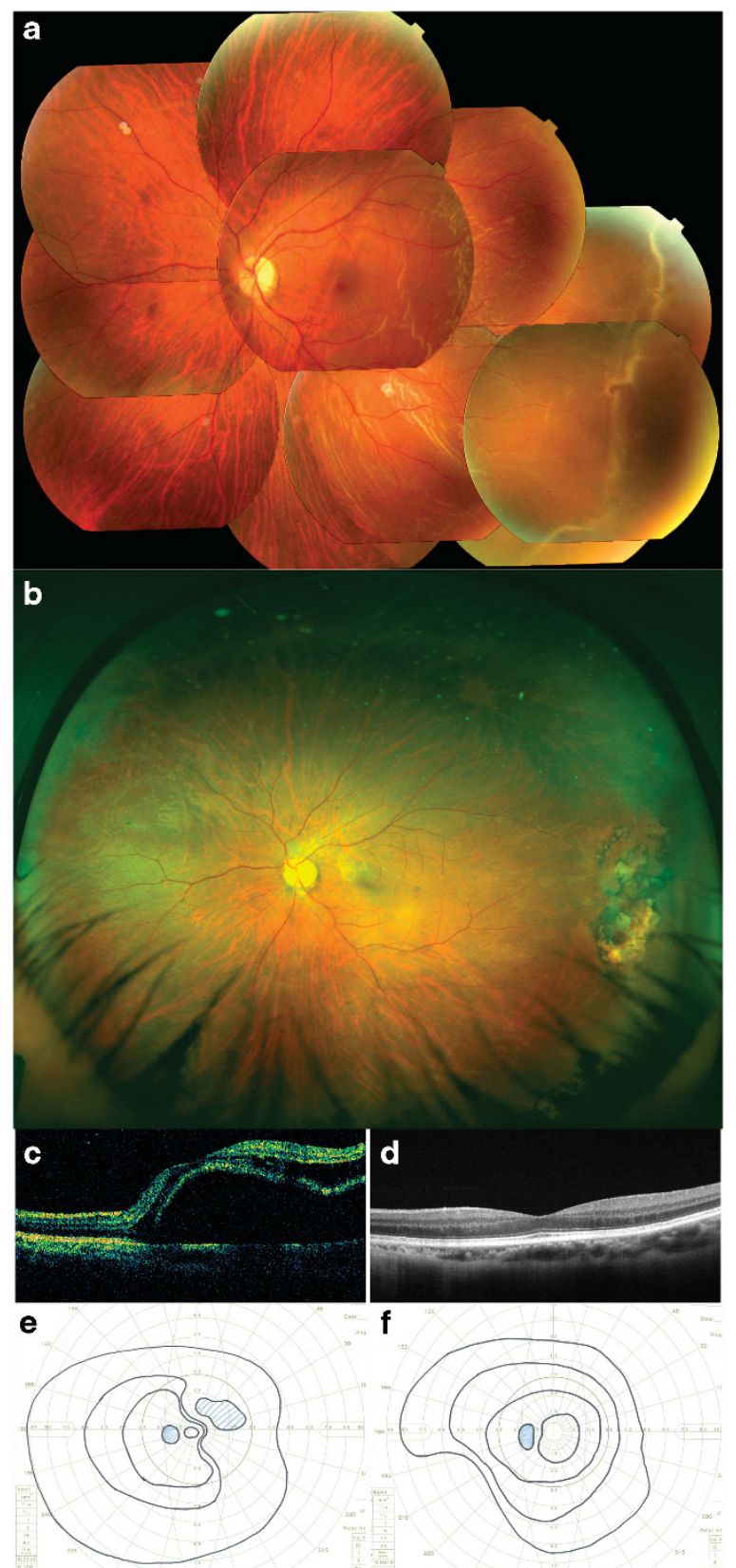

Figure 1 Fundus photographs, optical coherence tomography images, and Goldmann perimetry results from case 3. Fundus photographs of the left eye preoperatively (a) and 9 years postoperatively (b). Horizontal optical coherence tomography images of the left eye through the macula preoperatively (c) and 9 years postoperatively (d). Results of Goldmann perimetry of the left eye preoperatively (e) and 9 years postoperatively (f).

atrophy (case 2) were observed, both of which were present preoperatively.

The mean preoperative BCVA was 20/97 (range, 20/222-20/22); one eye had a BCVA of 20/40 or better. The mean postoperative BCVA 1,3 , and 6 months and 9 years postoperatively were 20/60 (range, 20/286-20/29),
20/45 (range, 20/222-20/22), 20/42 (range, 20/22220/22), and 20/33 (range, 20/133-20/20), respectively. At the final visit, three eyes had a BCVA of 20/40 or better.

All eyes had a transient IOP elevation several days postoperatively. Cases 1, 2, and 3 had a postoperative IOP higher than $40 \mathrm{~mm} \mathrm{Hg}$, and all four eyes were managed with antiglaucoma medications. The IOP spikes lasted no longer than 2 weeks and no eye required surgery to control the IOP. We observed mild postoperative intraocular inflammation, which typically follows cataract surgery and vitrectomy.

Goldmann visual field examinations performed 9 years postoperatively showed visual field defects that did not correspond to the locations of the detached retinas in all four eyes. These included an inferonasal defect in two eyes (cases 1 and 2), an inferotemporal defect in two eyes (cases 3 and 4), and a mild concentric constriction in one eye (case 1).

The ERG a- and b-wave amplitudes of the operated eyes were calculated as relative ratios to those of the fellow eyes. The mean a-wave amplitude ratio of the operated eyes to the fellow eyes was 0.68 preoperatively and 0.879 years postoperatively. The mean b-wave amplitude ratio of the operated eyes to the fellow eyes was 0.64 preoperatively and 0.929 years postoperatively.

Specular microscopy showed that the mean corneal endothelial cell density was 2365 cells $/ \mathrm{mm}^{2}$ preoperatively and 2592 cells $/ \mathrm{mm}^{2} 9$ years postoperatively.

\section{Discussion}

In this case series, we could cover the retinal breaks with Seprafilm in four eyes with a RRD. Successful retinal reattachment and substantial visual recovery were achieved with a single surgery in all four eyes. There were no immediate or late adverse events, and no eyes developed PVR postoperatively. Electroretinography and specular microscopy showed no apparent evidence of toxicity of Seprafilm 9 years postoperatively.

The three major methods currently used to reattach the retina are PPV, scleral buckling, and pneumatic retinopexy. ${ }^{8}$ In these vitreoretinal surgeries, retinal breaks are surrounded by application of cryopexy or laser photocoagulation. Regardless of the methods, the retinal breaks are not actually closed. The passage of intravitreal fluid through the retinal breaks into the subretinal space can be prevented permanently when scars form around the retinal breaks; however, the exposed retinal pigment epithelium within the retinal breaks may permit intravitreal migration of retinal pigment epithelial cells. Although Seprafilm was absorbed completely after a certain period of time, Seprafilm might act as a temporal 
barrier that covers retinal breaks until scars form around the retinal breaks.

One of the disadvantages of repairing RRDs with PPV and gas tamponade is the need for postoperative head positioning. In addition, intraocular gas tamponade cannot directly support inferior retinal breaks without strict prone positioning postoperatively. ${ }^{9}$ Seprafilm may obviate the need for this postoperative head positioning, which is often difficult and uncomfortable for patients. In the current case series, two of the four eyes (cases 1 and 2) had a RRD with inferior retinal breaks. We delivered Seprafilm into the eye after fluid-air exchange because the adhesive strength of Seprafilm decreases when it is wet. Although air was used as an internal tamponade, the patients were not instructed to maintain a prone position postoperatively. Covering the retinal breaks with Seprafilm kept the retina in place until laser scars developed.

One technical difficulty was achieving safe and effective delivery of the Seprafilm sheet into the eye. It took time to apply Seprafilm over the retinal breaks after fluid-air exchange in the first two operated eyes; however, the surgeon became more adept at delivering the Seprafilm sheet with experience. We think that the decreased fundus visibility during Seprafilm insertion can be now overcome if we use a wide-angle viewing system and chandelier illumination, which provides an excellent view of the peripheral retinal breaks even after fluid-air exchange. ${ }^{10}$ In the current case series, we delivered a $5 \times 2 \mathrm{~mm}$ sheet of Seprafilm through a $3 \mathrm{~mm}$ sclerotomy site. However, we need to develop a more efficient system for intraocular delivery of larger sheets of Seprafilm from smaller sclerotomy sites for 23,25 , or 27 gauge vitrectomy currently the worldwide standard.

The most common postoperative complication in the current series was ocular hypertension. All four eyes had transient IOP elevations several days postoperatively, which returned to normal within 2 weeks after onset. Although the exact mechanisms by which Seprafilm causes transient ocular hypertension are unclear, increased resistance to the flow of aqueous humor may occur in the trabecular meshwork as reported for ocular hypertension related to viscoelastic substances. ${ }^{11}$

We detected visual field defects either in the inferonasal or inferotemporal quadrant that did not correspond to the area of the RRDs. The visual field defects were more prominent in the first two operated eyes, in which it took longer to deliver the Seprafilm after fluid-air exchange. We speculated that these visual field defects were most likely due to mechanical or drying damage to the retina or optic nerve during the prolonged insertion procedures under air. ${ }^{12,13}$ As we did not observe any relationship between the location of Seprafilm placed and the visual field defects, we believe that this complication likely was secondary to mechanical insults related to the surgeries rather than the effects of the Seprafilm itself.

Biocompatible tissue adhesives have been tested previously to treat retinal detachments but were abandoned because of insufficient clinical efficacy or complications. ${ }^{14-18}$ For example, cyanoacrylate forms a hard mass rather than a thin sheet, which makes it inadequate for covering retinal breaks along with some toxicity. Furthermore, the long-term safety and visual outcomes of the tissue adhesives have not been investigated. In the current case series, all four patients were further evaluated to determine the long-term safety of Seprafilm. Seprafilm seems to be an appropriate material to patch retinal breaks as the visual outcomes obtained 9 years postoperatively showed no apparent adverse effects of intraocular application of the material.

The limitations of this study included the small sample size and lack of a control group. Therefore, we could not determine whether the anatomic success of the RRDs including the possible prevention of PVR was due to Seprafilm, and further studies are needed to validate the results. Although the outcome measures were predetermined and the data were gathered prospectively up to 6 months postoperatively, the evaluation of the long-term safety performed 9 years postoperatively were retrospective. Nevertheless, this study showed that covering retinal breaks with Seprafilm may promote retinal reattachment without gas tamponade and postoperative head positioning. In addition, the visual outcomes 9 years postoperatively showed no apparent adverse effects of intraocular application of Seprafilm. This study provided the basis for further clinical studies of retinal patching surgery in patients with RRDs.

\section{Summary}

\section{What was known before}

- Seprafilm adheres well to the retina in vitro. Seprafilm promotes retinal reattachment in an animal model of retinal detachment.

\section{What this study adds}

- Seprafilm may promote retinal reattachment without gas tamponade and postoperative head positioning in humans. The visual outcomes 9 years postoperatively showed no apparent adverse effects of intraocular application of Seprafilm.

\section{Conflict of interest}

The authors declare no conflict of interest.

\section{Acknowledgements}

This study was funded by Novartis Pharma. 


\section{References}

1 Khan MA, Brady CJ, Kaiser RS. Clinical management of proliferative vitreoretinopathy: an update. Retina 2015; 35: 165-175.

2 Tosi GM, Marigliani D, Romeo N, Toti P. Disease pathways in proliferative vitreoretinopathy: an ongoing challenge. J Cell Physiol 2014; 229: 1577-1583.

3 Gilbert CE, Grierson I, McLeod D. Retinal patching: a new approach to the management of selected retinal breaks. Eye 1989; 3: 19-26.

4 Becker JM, Dayton MT, Fazio VW, Beck DE, Stryker SJ, Wexner SD et al. Prevention of postoperative abdominal adhesions by a sodium hyaluronate-based bioresorbable membrane: a prospective, randomized, double-blind multicenter study. J Am Coll Surg 1996; 183: 297-306.

5 Diamond MP. Reduction of adhesions after uterine myomectomy by Seprafilm membrane (HAL-F): a blinded, prospective, randomized, multicenter clinical study. Seprafilm Adhesion Study Group. Fertil Steril 1996; 66: 904-910.

6 Sueda J, Sakuma T, Nakamura H, Usumoto N, Okuno T, Arai $\mathrm{M}$ et al. In vivo and in vitro feasibility studies of intraocular use of seprafilm to close retinal breaks in bovine and rabbit eyes. Invest Ophthalmol Vis Sci 2006; 47: 1142-1148.

7 Teruya K, Sueda J, Arai M, Tsurumaru N, Yamakawa R, Hirata A et al. Patching retinal breaks with Seprafilm in experimental rhegmatogenous retinal detachment of rabbit eyes. Eye 2009; 23: 2256-2259.

8 D'Amico DJ. Clinical practice. Primary retinal detachment. N Engl J Med 2008; 359: 2346-2354.
9 Tanner V, Minihan M, Williamson TH. Management of inferior retinal breaks during pars plana vitrectomy for retinal detachment. Br J Ophthalmol 2001; 85: 480-482.

10 Chalam KV, Shah VA. Optics of wide-angle panoramic viewing system-assisted vitreous surgery. Surv Ophthalmol 2004; 49: 437-445.

11 Harooni M, Freilich JM, Abelson M, Refojo M. Efficacy of hyaluronidase in reducing increases in intraocular pressure related to the use of viscoelastic substances. Arch Ophthalmol 1998; 116: 1218-1221.

12 Welch JC. Dehydration injury as a possible cause of visual field defect after pars plana vitrectomy for macular hole. Am J Ophthalmol 1997; 124: 698-699.

13 Yan H, Dhurjon L, Chow DR, Williams D, Chen JC. Visual field defect after pars plana vitrectomy. Ophthalmology 1998; 105: 1612-1616.

14 McCuen BW, Hida T, Sheta SM. Transvitreal cyanoacrylate retinopexy in the management of complicated retinal detachment. Am J Ophthalmol 1987; 104: 127-132.

15 Hida T, Sheta SM, Proia AD, McCuen BW. Retinal toxicity of cyanoacrylate tissue adhesive in the rabbit. Retina 1988; 8: 148-153.

16 Sheta SM, Hida T, McCuen BW. Cyanoacrylate tissue adhesive in the management of recurrent retinal detachment caused by macular hole. Am J Ophthalmol 1990; 109: 28-32.

17 Hartnett ME, Hirose T. Cyanoacrylate glue in the repair of retinal detachment associated with posterior retinal breaks in infants and children. Retina 1998; 18: 125-129.

18 Hotta K, Hirakata A, Hida T. The management of retinal detachments associated with choroidal colobomas by vitrectomy with cyanoacrylate retinopexy. Jpn J Ophthalmol 1998; 42: 323-326.

Supplementary Information accompanies this paper on Eye website (http://www.nature.com/eye) 